\title{
Factors Associated with Uptake of HIV Test Results in a Nationally Representative Population-Based AIDS Indicator Survey
}

\author{
${ }^{I}$ U.S. Centers for Disease Control and Prevention, Nairobi, Kenya \\ ${ }^{2}$ San Francisco Department of Public Health, California, USA \\ ${ }^{3}$ John Hopkins Bloomberg School of Public Health, Baltimore, USA \\ ${ }^{4}$ National AIDS/STD Control Programme, Nairobi, Kenya \\ ${ }^{5}$ Kenya Medical Research Institute, Nairobi, Kenya \\ ${ }^{6}$ U.S. Centers for Disease Control and Prevention, Atlanta, USA
}

Mary Mwangi ${ }^{*}, 1$, Timothy A. Kellogg ${ }^{2}$, Sufia S. Dadabhai ${ }^{3}$, Rebecca Bunnell ${ }^{1}$, Godfrey Baltazar ${ }^{4}$, Carol Ngare ${ }^{4}$, George K'Opiyo ${ }^{5}$ Margaret Mburu ${ }^{1}$ and Andrea A. Kim ${ }^{1,6}$

\begin{abstract}
Population-based surveys with HIV testing in settings with low testing coverage provide opportunities for participants to learn their HIV status. Survey participants (15-64 years) in a 2007 nationally representative populationbased HIV serologic survey in Kenya received a voucher to collect HIV test results at health facilities 6 weeks after blood draw. Logistic regression models were fitted to identify predictors of individual and couple collection of results. Of 15,853 adults consenting to blood draw, 7,222 (46.7\%) collected HIV test results $(46.5 \%$ men, $46.8 \%$ women). A third (39.5\%) of HIV-infected adults who were unaware of their infection and $48.2 \%$ of those who had never been tested learned their HIV status during KAIS. Individual collection of HIV results was associated with older age, with the highest odds among adults aged 60-64 years (adjusted odds ratio [AOR], 1.6, 95\% confidence interval [CI] 1.2-2.1); rural residence (AOR 1.8, 95\%CI 1.2-2.6); and residence outside Nairobi, with the highest odds in the sparsely populated North Eastern province (AOR 8.0, 95\%CI 2.9-21.8). Of 2,685 married/cohabiting couples, $18.5 \%$ collected results as a couple. Couples in Eastern province and in the second and middle wealth quintiles were more likely to collect results than those in Nairobi (AOR 3.2, 95\%CI 1.1-9.4) and the lowest wealth quintile (second AOR 1.5, 95\%CI 1.1-2.3; middle AOR 1.6, 95\% CI 1.2-2.3, respectively. Many participants including those living with HIV learned their HIV status in KAIS. Future surveys need to address low uptake of results among youth, urban residents, couples and those with undiagnosed HIV infection.
\end{abstract}

Keywords: AIDS indicator survey, HIV, HIV test results, Kenya, population-based.

\section{INTRODUCTION}

Many countries with generalized HIV epidemics routinely conduct nationally-representative, populationbased surveys [e.g., Demographic and Health Surveys (DHS) or AIDS Indicator Surveys (AIS)] with an HIV testing component to quantify HIV prevalence, plan prevention, care and treatment services and evaluate the national HIV program $[1,2]$. In recent years, discourse on the ethics of HIV testing in population-based surveys has highlighted the right of survey participants to learn their HIV status in order to access increasingly available HIV health services [3, 4]. These arguments stem from the belief that research and survey participants have rights to appropriate information before, during and after studies to help them make informed choices and access appropriate services [5].

*Address correspondence to this author at the Division of Global HIV/AIDS, Centers of Disease Control and Prevention, Mbagathi Way, P.O. Box 60600621 Village Market, Nairobi, Kenya; Tel: +254 2722203718 ; Fax: +254 20271 4745; E-mail: mmwangi@ke.cdc.gov
In 2009, a UNAIDS/WHO consultation on Ethics in HIV Surveillance recommended use of HIV surveillance approaches that maximize beneficial outcomes for individuals and communities [6]. Additionally, countries were advised to make informed decisions on how best to balance the benefits and risks of returning HIV and other test results to participants. In 1995, the United States (U.S.) discontinued unlinked anonymous testing (UAT) for HIV infection among mothers and infants due to availability of HIV treatment and subsequently ended funding of all UAT surveys of HIV [7].

In developing countries, reporting biological results to survey participants needs to be balanced against several factors, including where tests are performed, social meaning of the disease, the physical and social contexts in which reporting occurs and the health care delivery system [4]. Past surveys in Kenya and elsewhere have used several approaches to help survey participants learn their HIV status. These approaches include referral for free voluntary counseling and testing (VCT) services, mobile VCT, and travel reimbursement to HIV testing sites [2, 8]. In the 2003 
Kenya DHS, participants were either referred to the nearest VCT site or to a mobile VCT site in regions with no VCT services to learn their HIV status [9]. The survey did not effectively measure uptake of VCT services among survey participants, however, available data indicate that females (15-49 years) who accessed VCT had a higher HIV prevalence $(13 \%)$ compared to the national female HIV prevalence $(9 \%)$ while male participants who accessed VCT had HIV prevalence similar to the national male HIV prevalence $(5 \%)$.

Surveys that have directly returned results to participants have done so either in the home at the time of the interview [10] or they have made results available in designated health facilities after centralized laboratory testing [11]. In rural southwestern Uganda, rapid home-based HIV testing and counseling (HBTC) of population-based cohort study participants in selected clusters in a population-based serosurvey resulted in a significant three-fold (37\%) increase of HIV testing and counseling compared to an uptake of less than $10 \%$ in previous surveys [12]. The 2007 Kenya AIDS Indicator Survey (KAIS) used a facility-based approach to inform participants of their HIV test results [11]. To inform planning of future surveys, we report uptake and describe the characteristics of individuals and couples who collected HIV test results in the 2007 KAIS and examine factors associated with individual and couple collection of results.

\section{METHODS}

The 2007 KAIS was a cross-sectional, two stage cluster sampling survey of Kenyan adults aged 15-64 years designed to provide national and provincial estimates of sociodemographic, behavioral, and biologic correlates of HIV infection. In addition to a structured questionnaire, venous blood samples were drawn from consenting participants for HIV antibody testing (Vironostika HIV-1/2 antigen/antibody EIA for screening, Murex HIV.1.2.O EIA for confirmation, with discrepancies resolved by Roche Amplicor HIV DNA PCR v1.5). For participants who could not provide venous blood, dried blood spots were collected for HIV testing (Vironostika HIV UNIFORM II Plus O v 3.3 and Murex HIV 1.2.0). Samples were tested at the Kenya National HIV Reference Laboratory and underwent quality assurance testing at the Kenya Medical Research Institute laboratory. Final HIV test results were recorded by laboratory staff on a paper form using a participant's unique study identification number and delivered to selected health facilities in each survey cluster.

\section{Collection of Test Results}

Participants were encouraged by the survey teams to collect test results with their spouse or sexual partner and informed that results would be available approximately 6 weeks after the blood draw. Results were available at two facilities; a health facility within the study cluster and one outside the cluster to provide an option for those concerned about confidentiality at facilities in the study cluster. Survey teams issued a test results voucher to participants who consented to blood draw. The voucher had a unique study identification number and sex of the participant; the facility name, location and operating hours; and beginning and end dates when results would be available.
A team of 202 trained and experienced counselors provided results and counseling to participants. Counselors were trained on clinical aspects of HIV prevention and treatment of HIV and STIs, partner testing and disclosure, basic counseling techniques and the approved protocol for returning results to participants. Participants with valid vouchers received test results and counseling in a private room in the health facility. Couples were first counseled individually and upon informed consent from both parties, counseled as a couple. Participants received standard counseling messages and informational brochures on HIV prevention, care and treatment of HIV and STIs and condoms. Counselors used a facility directory to refer participants for follow-up services as needed. Participants with non-reactive results were counseled on prevention and reminded that test results represented their status at the time of sample collection. After each counseling session, counselors recorded the participant's sex, whether the participant received results individually or with a partner, and referrals on the counselor results form. Participants were given a period of one-month to collect test results, after which all results forms were returned to the National AIDS and STI Control Program for data entry.

\section{Data Collection and Statistical Analysis}

Data from results forms were double-entered into EPI Info Version 3.0 (U.S. Centers for Disease Control and Prevention) and analyzed using SAS version 9.2 (SAS Institute Inc, Cary North Carolina, USA). All analyses were weighted and adjusted for complex survey design. Bivariate and multivariate analyses were conducted to examine differences in results uptake by socio-demographic, behavioral and serological characteristics. The analyses included proportions, odds ratios (OR), adjusted odds ratios (AOR) and 95\% confidence intervals (CI). We used a RaoScott chi-square test to evaluate the association between uptake of HIV results and categorical variables. Variables significantly associated with the outcomes of interest (individual collection of results and collection of results as a couple) with $\mathrm{p}<0.2$ in the bivariate analysis were included in logistic regression models. Variables that remained associated with the outcome in the final model at a significance level of $p<0.05$ were considered independent predictor variables.

A secondary analysis was conducted among KAIS participants who identified as the spouse or co-habiting partner of another KAIS participant to assess rates of test collection among married and cohabiting couples. We excluded couples in which a KAIS participant collected results with a non-KAIS partner. Each respondent within the couple was evaluated separately to determine whether they had collected results with their partner or individually. We describe the socio-demographic, behavioral, knowledge and serological characteristics of couples that collected HIV test results and assess factors associated with receiving results as a couple.

\section{Ethics}

The survey protocol was approved by the Ethical Review Committee at the Kenya Medical Research Institute (KEMRI) and the Institutional Review Board at the U.S. Centers for Diseases Control and Prevention (CDC). Survey 
participants provided separate verbal informed consent to participate in the interview, blood draw and for storage of their blood specimens for future testing.

\section{RESULTS}

\section{Socio-Demographic Characteristics of Participants who Collected HIV Test Results}

Overall the median number of days between blood sample collection and results collection was 67days (9.6 weeks). Unexpected delays in the laboratory, health facilities and displacement of participants and counselors due to the 2007-2008 post-election violence delayed delivery of results in a small number of study clusters.

Of 15,853 participants who consented to blood draw, $7,222(46.7 \%)$ collected their test results $(46.5 \%$ of men, $46.8 \%$ of women, $p=0.835$ ) (Table 1). Individual collection of results increased with age with uptake ranging from $41.3 \%$ (ages $25-29$ ) to $59.6 \%$ (ages $60-64$ ), $\mathrm{p}<0.001$. Adults who had never been married or cohabited had the lowest uptake of tests results (41.4\%) compared with those currently married or cohabitating $(48.9 \%)$ or widowed $(53.5 \%), p<0.001]$. Rural residents were nearly two times more likely to collect results $(52.7 \%)$ than urban residents $(26.9 \%, \mathrm{p}<0.001)$; collection of test results was highest in the sparsely populated North Eastern province $(77.7 \%)$ and lowest in Nairobi, the capital city $(13.9 \%), \mathrm{p}<0.001$.

\section{HIV Testing, Knowledge of HIV Status, Serological and Behavioral Characteristics}

Overall, HIV prevalence among those who collected their results was $6.1 \%(95 \%$ CI 5.3-7.0) compared with $7.9 \%$ (95\% CI 7.1-8.8), $(\mathrm{p}=0.002)$ among those who did not collect results (data not shown). Among HIV-infected adults, uptake was $40.3 \%$ compared to $47.2 \%$ among those with no HIV infection $(p=0.002)$, irrespective of whether they knew their HIV status or not. Adults who expressed willingness to be tested for HIV at home were more likely to collect results than those not willing to be tested at home (46.8\% vs $43.1 \%$, $\mathrm{p}=0.041$ ); however, $87.2 \%$ of adults who did not collect their HIV results expressed willingness to be tested for HIV at home (data not shown).

Participants who had never been tested for HIV or had ever been tested but did not receive their last HIV test results were more likely to collect results $(48.2 \%)$ than those who had been tested previously and received their last HIV test result $(42.8 \%), p<0.001$. More than a third $(39.5 \%)$ of HIVinfected adults who had incorrect knowledge of their HIV status and close to half of HIV-uninfected adults (48.7\%) who did not know their HIV status collected their results.

Overall, sexually-active adults who reported unprotected last sex with a partner of unknown or known discordant HIV status in the past year were more likely to collect their results than those who reported using a condom at last sex e (48.4\% vs $38.0 \%, \mathrm{p}<0.001)$. Nevertheless, sexually-active women who reported unprotected last sex with a partner of unknown or known discordant HIV status in the past year were more likely to collect results $(48.6 \%)$ compared to men who reported unprotected sex with a partner of unknown or known discordant HIV status in the past year $(35.8 \%$, $\mathrm{p}<0.001$, data not shown). Participants with accepting attitudes towards people with HIV/AIDS were less likely to collect results compared to those with low acceptance of people with HIV/AIDS (43.6\% vs 49.3\%, p < 0.001).

In multivariate analysis (Table 2), the odds of individual collection of results (Table 2) increased significantly with age compared to the youngest age group (15-24 years), with the highest odds among adults aged 60-64 years (AOR 1.6, $95 \%$ CI 1.2-2.1). Collection of results was significantly higher among persons living in rural areas (AOR 1.8, 95\% CI 1.2-2.6), residence in all provinces compared to Nairobi (see Table 2 for AORs, $p<0.001$ ) and among persons who reported willingness to be tested for HIV at home (AOR 1.2, 95\%CI 1.0-1.4). Moreover, HIV-infected adults who believed they were HIV-negative based on their last HIV test and those who had never been tested for HIV were significantly less likely to collect results (AOR 0.8 , 95\% CI 0.6-0.9) compared to those who correctly knew their HIV status.

\section{Collection of Results Among Couples}

Of the 9,691 households sampled, 2,752 married or cohabiting couples (head of household and his/her primary partner) completed interviews and consented to blood draw [11]. We excluded 67 couples either because one partner collected results with a non-KAIS partner $(n=60)$ or because of missing information on how they collected results $(n=7)$. Overall, $18.5 \%$ of 2,685 KAIS couples collected results as a couple (Table 3); $44.4 \%$ had either one or both partners collect results individually; and for $37.1 \%$ of couples, both partners did not collect results (data not shown).

Couples living in rural areas were more likely to collect results together compared to those in urban areas $(20.3 \% v s$ $9.0 \%, \mathrm{p}<0.001)$. Couples in Eastern province had the highest test result uptake $(26.9 \%)$ while those in Nairobi had the lowest $(5.1 \%)$. Couples in the second $(22.3 \%)$, middle $(23.2 \%)$ and fourth $(20.2 \%)$ wealth quintiles were twice as likely to collect results compared to those in the highest wealth quintile $(10.8 \%)$.

There was no significant difference in collection of results by prior HIV testing history, awareness of VCT, knowledge of HIV discordance and knowledge of couple HIV status (Table 3). Of HIV discordant couples who did not know their HIV status, $22.3 \%$ collected test results. Similarly, only $(15.7 \%)$ of the 74 HIV concordant positive couples who did not know their HIV status collected results.

In multivariate analysis, collection of results among couples was significantly associated with living in Eastern province (AOR 4.8, 95\% CI 2.0-11.5) compared to residence in Nairobi province; and being in the second (AOR 1.5, 95\% CI 1.1-2.1) and middle (AOR 1.5, 95\% CI 1.1-2.1) wealth quintiles compared to the lowest wealth quintile (Table 4).

\section{DISCUSSION}

Returning HIV test results through health facilities in the 2007 KAIS resulted in close to half of the survey participants learning their HIV status, higher than the national uptake of lifetime HIV testing of $33 \%$ found in KAIS [11]. Moreover, $42.8 \%$ of all participants who had never been tested for HIV 
Table 1. Characteristics of Men and Women (Age 15-64 Years) who Collected HIV Test Results in the 2007 Kenya AIDS Indicator Survey

\begin{tabular}{|c|c|c|c|c|c|}
\hline Characteristic & Total & No. Collected Results & Weighted \% & $95 \%$ CI & p-Value \\
\hline Total & 15,853 & 7222 & 46.7 & $(43.8-49.5)$ & -- \\
\hline \multicolumn{6}{|l|}{ Sex } \\
\hline Male & 6804 & 3058 & 46.5 & $(43.4-49.7)$ & 0.835 \\
\hline Female & 9049 & 4164 & 46.8 & $(43.8-49.7)$ & \\
\hline \multicolumn{6}{|l|}{ Age group } \\
\hline $15-24$ & 5135 & 2114 & 42.8 & $(39.5-46.1)$ & $<.0001$ \\
\hline $25-29$ & 2219 & 907 & 41.3 & $(37.9-44.8)$ & \\
\hline $30-39$ & 3554 & 1603 & 45.7 & $(42.4-48.9)$ & \\
\hline $40-49$ & 2599 & 1327 & 53.7 & $(49.7-57.8)$ & \\
\hline $50-59$ & 1749 & 915 & 52.6 & $(48.4-56.8)$ & \\
\hline $60-64$ & 597 & 356 & 59.6 & $(54.7-64.5)$ & \\
\hline \multicolumn{6}{|l|}{ Marital Status } \\
\hline Never married & 4523 & 1786 & 41.4 & $(38.2-44.7)$ & $<.0001$ \\
\hline Separated/divorced & 919 & 393 & 43.6 & $(38.7-48.4)$ & \\
\hline Widowed & 772 & 403 & 53.5 & $(48.3-58.6)$ & \\
\hline Married/cohabiting & 9639 & 4640 & 48.9 & $(45.8-52.0)$ & \\
\hline \multicolumn{6}{|l|}{ Residence } \\
\hline Urban & 3922 & 962 & 26.9 & $(20.9-32.8)$ & $<.0001$ \\
\hline Rural & 11,931 & 6260 & 52.7 & $(49.3-56.0)$ & \\
\hline \multicolumn{6}{|l|}{ Province } \\
\hline Nairobi & 1811 & 275 & 13.9 & $(10.2-17.6)$ & $<.0001$ \\
\hline Central & 2277 & 999 & 43.6 & $(35.8-51.3)$ & \\
\hline Coast & 1773 & 728 & 42.9 & $(34.3-51.5)$ & \\
\hline Eastern & 2553 & 1479 & 59.8 & $(52.7-67.0)$ & \\
\hline North Eastern & 753 & 557 & 77.7 & $(64.3-91.1)$ & \\
\hline Nyanza & 2380 & 1060 & 45.4 & $(37.5-53.3)$ & \\
\hline Rift Valley & 2268 & 1068 & 49.5 & $(42.9-56.2)$ & \\
\hline Western & 2038 & 1056 & 50.8 & $(42.9-58.6)$ & \\
\hline \multicolumn{6}{|l|}{ Education } \\
\hline No education & 2214 & 1291 & 56.3 & $(50.3-62.3)$ & $<.0001$ \\
\hline Incomplete primary & 4499 & 2220 & 50.6 & $(46.8-54.4)$ & \\
\hline Complete primary & 3802 & 1747 & 47.9 & $(44.4-51.5)$ & \\
\hline Secondary + & 5338 & 1964 & 39.2 & $(35.8-42.6)$ & \\
\hline \multicolumn{6}{|l|}{ Wealth Index ${ }^{1}$} \\
\hline Lowest & 2758 & 1570 & 56.1 & $(50.0-62.3)$ & $<.0001$ \\
\hline Second & 2913 & 1555 & 53.0 & $(48.6-57.3)$ & \\
\hline Middle & 3049 & 1563 & 52.2 & $(48.1-56.3)$ & \\
\hline Fourth & 3095 & 1364 & 46.2 & $(42.0-50.3)$ & \\
\hline Highest & 4038 & 1170 & 32.9 & $(27.8-37.9)$ & \\
\hline
\end{tabular}




\begin{tabular}{|c|c|c|c|c|c|}
\hline Characteristic & Total & No. Collected Results & Weighted \% & $95 \% \mathrm{CI}$ & p-Value \\
\hline \multicolumn{6}{|c|}{ Accepting attitudes towards people with HIV/AIDS ${ }^{2}$} \\
\hline No & 7610 & 3685 & 49.3 & $(46.1-52.5)$ & \\
\hline \multicolumn{6}{|l|}{ Heard of $\mathrm{VCT}^{3}$} \\
\hline No/Don't Know/Unsure & 2061 & 1169 & 55.0 & $(49.6-60.3)$ & \\
\hline \multicolumn{6}{|c|}{ Willingness to be tested for HIV at home ${ }^{3}$} \\
\hline Yes & 13,550 & 6,102 & 46.8 & $(43.9-49.8)$ & 0.0410 \\
\hline No/Don't know/Unsure & 1930 & 832 & 43.1 & $(39.1-47.1)$ & \\
\hline No/Never received results & 10,236 & 4797 & 48.2 & $(45.2-51.1)$ & \\
\hline \multicolumn{6}{|l|}{ HIV status ${ }^{4}$} \\
\hline HIV-negative & 14,723 & 6802 & 47.2 & $(44.3-50.1)$ & .0021 \\
\hline HIV-positive & 1104 & 411 & 40.3 & $(35.4-45.2)$ & \\
\hline \multicolumn{6}{|l|}{ Knowledge of HIV status ${ }^{4}$} \\
\hline Correct knowledge of HIV status & 4728 & 1942 & 43.0 & $(39.5-46.5)$ & $<.0001$ \\
\hline Incorrect knowledge, HIV infected & 913 & 327 & 39.5 & $(34.5-44.5)$ & \\
\hline Incorrect knowledge, HIV uninfected & 9637 & 4577 & 48.7 & $(45.7-51.7)$ & \\
\hline
\end{tabular}

${ }^{1}$ A composite measure of the living standards of a household, based on household ownership of selected assets, materials used for houshing construction, access to water and sanitation. Households in the sample are placed on a continuous scale of relative wealth using principal components analysis. Individuals are ranked according to the household score and the sample is divided into five groups with an equal number of indiviuals (quintiles).

${ }^{2}$ Based on a composite score derived from responses to four questions on attitudes towards people living with HIV/AIDS. Analysis excludes 373 respondents who had never heard of AIDS.

${ }^{3}$ Excludes 373 adults who reported they had never heard of AIDS.

${ }^{4}$ Excludes 26 adults with indeterminate HIV results.

${ }^{5}$ Includes participants who reported sexual activity in the past 12 months; excludes 5 adults with missing information on sexual behavior.

${ }^{6}$ Includes HIV-infected adults who had unprotected sex with a known concordant HIV positive partner.

Participants with no education and those in the lowest wealth quintile had the highest collection rates $(56.3 \%$ and $56.1 \%$, respectively) while those with secondary or higher education and in the highest wealth quintile had the lowest uptake $(39.2 \%$ and $32.9 \%$, respectively).

and over a third $(39.4 \%)$ of those living with HIV but unaware due to never testing in the past collected their HIV test results. These findings demonstrate that in Kenya and other countries with similar HIV epidemics and social contexts, population-based surveys can contribute towards universal awareness of HIV status while still meeting a country's HIV surveillance needs.

Individual collection of HIV test results increased with age and was associated with rural residence, willingness to be tested for HIV at home and correct knowledge of HIV status. The high results collection rate among rural residents and those living outside Nairobi province could be attributed to the limited availability or accessibility of routine HIV testing services in rural areas compared to urban areas, including Nairobi. The KAIS survey may have presented a unique opportunity for participants living in underserved regions to learn their HIV status. Similarly, survey response rates were higher among households and residents in rural clusters than in urban clusters [11]. Additionally, our analysis identified segments of the surveyed population that are less likely to collect HIV test results through health facilities, highlighting the need to consider alternative approaches in future surveys that could increase uptake of results among the youth, urban residents and persons living with undiagnosed HIV infection.

The 2007 KAIS [11] found high acceptance of HIV HBTC nationally ( $83.5 \%$ among adults $15-64$ years). This finding and the high acceptance of HBTC among participants who did not collect their HIV test results in our analysis suggest that offering HBTC within a survey could increase knowledge of HIV status among survey participants.

We encouraged all survey participants to collect results with their sexual/marital partners. However, only two out of 
Table 2. Factors Associated with Individual Collection of HIV Test Results Among Men and Women (15-64 Years) in the 2007 Kenya AIDS Indicator Survey

\begin{tabular}{|c|c|c|c|c|}
\hline Characteristic & Unadjusted Odds Ratio (95\% CI) & p-Value & Adjusted Odds Ratio $\left(95 \%\right.$ CI) ${ }^{1}$ & p-Value \\
\hline \multicolumn{5}{|l|}{ Sex } \\
\hline Female & $1.01(0.93-1.10)$ & 0.8351 & $1.01(0.93-1.11)$ & 0.7860 \\
\hline \multicolumn{5}{|l|}{ Age group } \\
\hline $25-29$ & $0.94(0.84-1.06)$ & 0.2996 & $0.95(0.83-1.10)$ & 0.5104 \\
\hline $30-39$ & $1.12(1.00-1.26)$ & 0.0463 & $1.04(0.89-1.22)$ & 0.5887 \\
\hline $40-49$ & $1.55(1.38-1.75)$ & $<.0001$ & $1.36(1.14-1.63)$ & 0.0005 \\
\hline $50-59$ & $1.48(1.27-1.73)$ & $<.0001$ & $1.25(1.02-1.53)$ & 0.0293 \\
\hline Rural & $3.03(2.17-4.21)$ & $<.0001$ & $1.80(1.24-2.61)$ & 0.0020 \\
\hline \multicolumn{5}{|l|}{ Province } \\
\hline Nairobi & 1.0 & -- & 1.0 & -- \\
\hline Central & $4.78(3.08-7.44)$ & $<.0001$ & $2.55(1.48-4.40)$ & 0.0008 \\
\hline Coast & $4.65(2.91-7.43)$ & $<.0001$ & $2.93(1.70-5.04)$ & 0.0001 \\
\hline Eastern & $9.23(6.02-14.15)$ & $<.0001$ & $4.66(2.71-8.02)$ & $<.0001$ \\
\hline North Eastern & $21.58(9.40-49.53)$ & $<.0001$ & $7.98(2.92-21.79)$ & $<.0001$ \\
\hline Nyanza & $5.14(3.30-8.01)$ & $<.0001$ & $2.59(1.47-4.58)$ & 0.0010 \\
\hline \multicolumn{5}{|l|}{ Knowledge of HIV status } \\
\hline Correct knowledge of HIV status & 1.0 & -- & 1.0 & -- \\
\hline Incorrect knowledge, HIV- infected & $0.86(0.71-1.06)$ & 0.1549 & $0.75(0.61-0.93)$ & 0.0080 \\
\hline Incorrect knowledge, HIV uninfected & $1.26(1.12-1.41)$ & $<.0001$ & $0.94(0.84-1.04)$ & 0.2436 \\
\hline Not willing to disclose status & $1.73(1.11-2.69)$ & 0.0152 & $1.16(0.76-1.78)$ & 0.4792 \\
\hline
\end{tabular}

${ }^{1}$ Multivariate model controlled for all other variables in Table 2. Marital status, education, wealth index, accepting attitudes towards persons living with HIV/AIDS and unprotected last sex with a partner of unknown or known HIV discordant status in the past 12 months were included in the model and were insignificant.

ten married/cohabiting adults collected results as a couple. Similar proportions of couples who had never been tested for HIV and of those living with HIV but unaware of their status learned their HIV status during KAIS. Given that most new HIV infections in Kenya occur among heterosexual couples $[12,13]$, the national HIV/AIDS program has prioritized couples testing, in part by scaling up HBTC. Of note, the next KAIS survey will offer HBTC to all survey participants, a shift that is expected to reach more couples and other segments of the population that were less likely to collect results through health facilities. The logistics of this approach, including participant and household burden needs careful consideration.

The test results uptake in KAIS could have been influenced by several factors. Owing to the 6-week delay in returning HIV test results to health facilities and referrals provided during the survey, some participants could have sought VCT and saw no need to collect survey results. Further, unexpected delays in the central laboratory and in health facilities following the 2007-2008 post-election violence in Kenya could have negatively affected collection of test results in affected study clusters. 
Table 3. Socio-Demographic, Behavioral and Serological Characteristics of Married and Cohabiting Men and Women (15-64 Years) in the 2007 Kenya AIDS Indicator Survey who Collected HIV Test Results as a Couple

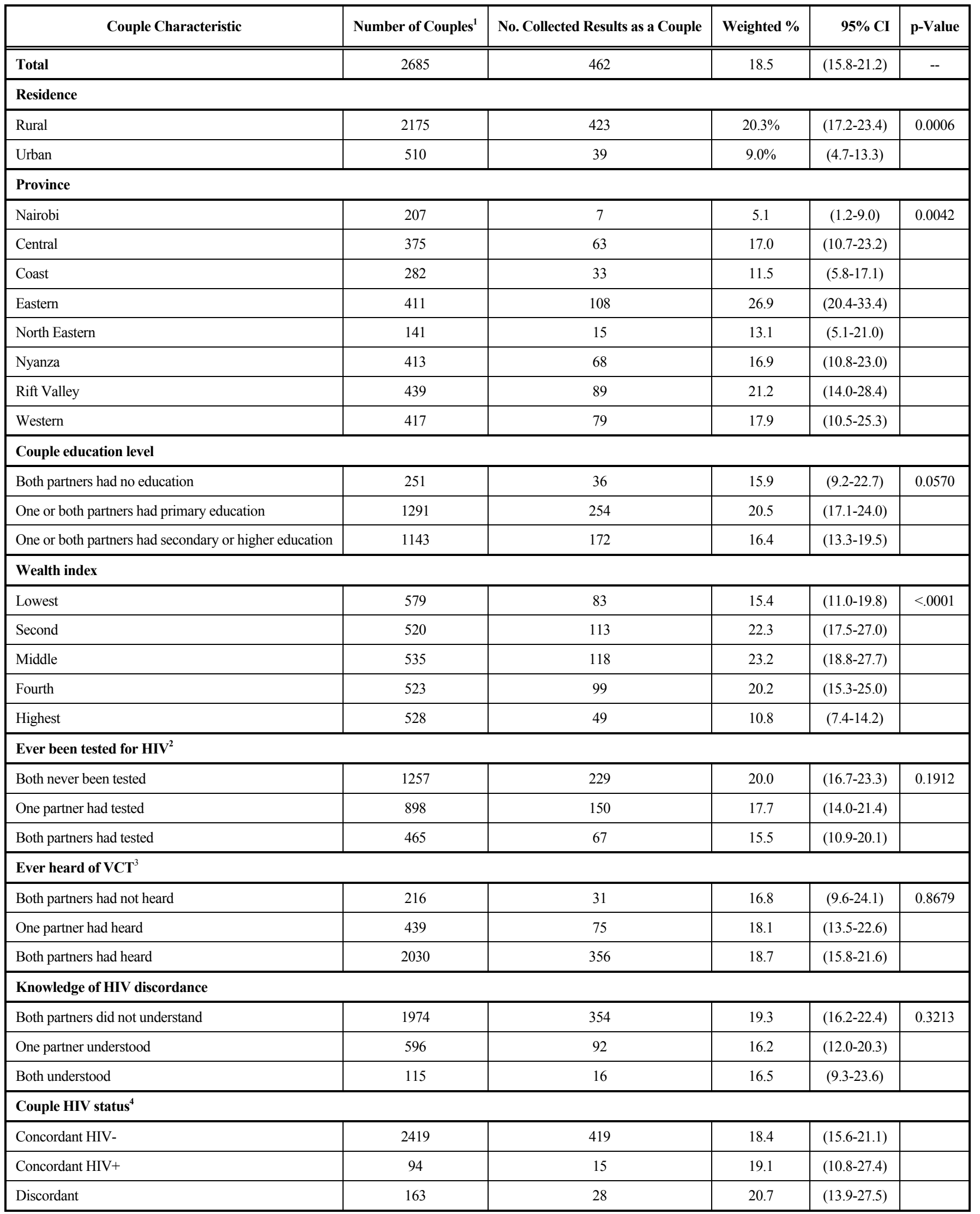


(Table 3) contd.....

\begin{tabular}{|c|c|c|c|c|c|}
\hline Couple Characteristic & Number of Couples ${ }^{1}$ & No. Collected Results as a Couple & Weighted \% & $95 \% \mathrm{CI}$ & p-Value \\
\hline \multicolumn{6}{|l|}{ Knowledge of couple HIV status ${ }^{4}$} \\
\hline Correctly knew status & 391 & 55 & 14.7 & $(10.1-19.2)$ & 0.2376 \\
\hline Don't know status, concordant negative & 1937 & 343 & 19.0 & $(16.0-22.0)$ & \\
\hline Don't know status, concordant positive & 74 & 10 & 15.7 & $(8.1-23.2)$ & \\
\hline Don't know status, discordant & 152 & 28 & 22.3 & $(15.2-29.4)$ & \\
\hline Not willing to disclose status & 122 & 26 & 21.1 & $(12.0-30.2)$ & \\
\hline
\end{tabular}

${ }^{1}$ Excludes 67 couples; 60 collected results with a non-KAIS partner and 7 had missing information.

${ }^{2}$ Excludes 65 couples with missing information on HIV testing.

${ }^{3}$ Adults who reported they had not heard of AIDS were classified as not having heard of VCT.

${ }^{4}$ Excludes 9 couples with indeterminate HIV results.

Table 4. Factors Associated with Collection of HIV Test Results as a Couple Among Married and Cohabiting Men and Women (15-64 Years) in 2007 Kenya AIDS Indicator Survey

\begin{tabular}{|c|c|c|c|c|}
\hline Couple Characteristic & Unadjusted OR (95\% CI) & p-Value & 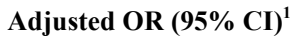 & p-Value \\
\hline \multicolumn{5}{|l|}{ Residence } \\
\hline Urban & 1.0 & -- & 1.0 & -- \\
\hline Rural & $2.59(1.48-4.52)$ & 0.0008 & $1.62(0.77-3.41)$ & 0.2012 \\
\hline \multicolumn{5}{|l|}{ Province } \\
\hline Nairobi & 1.0 & -- & 1.0 & -- \\
\hline Central & $3.80(1.52-9.51)$ & 0.0043 & $1.92(0.61-5.99)$ & 0.2625 \\
\hline Coast & $2.41(0.91-6.40)$ & 0.0770 & $1.46(0.46-4.58)$ & 0.5197 \\
\hline Eastern & $6.84(2.87-16.29)$ & $<.0001$ & $3.22(1.10-9.44)$ & 0.0328 \\
\hline North Eastern & $2.79(0.96-8.09)$ & 0.0583 & $1.74(0.48-6.35)$ & 0.3995 \\
\hline Nyanza & $3.78(1.52-9.43)$ & 0.0043 & $1.80(0.59-5.49)$ & 0.3002 \\
\hline Rift Valley & $5.00(2.01-12.44)$ & 0.0005 & $2.56(0.88-7.45)$ & 0.0845 \\
\hline Western & $4.06(1.58-10.48)$ & 0.0037 & $2.00(0.61-6.56)$ & 0.2547 \\
\hline \multicolumn{5}{|l|}{ Wealth index } \\
\hline Lowest & 1.0 & -- & 1.0 & -- \\
\hline Second & $1.58(1.08-2.30)$ & 0.0189 & $1.54(1.05-2.27)$ & 0.0275 \\
\hline Middle & $1.67(1.17-2.37)$ & 0.0046 & $1.64(1.16-2.32)$ & 0.0049 \\
\hline Fourth & $1.39(0.92-2.10)$ & 0.1171 & $1.44(0.94-2.21)$ & 0.0927 \\
\hline Highest & $0.67(0.42-1.06)$ & 0.0877 & $1.08(0.61-1.92)$ & 0.7948 \\
\hline
\end{tabular}

${ }^{1}$ Multivariate model controlled for all other variables in Table 4. Couple education level and knowledge of couple HIV status were included in the model and were not significant.

While the 6-week period allowed for centralized laboratory testing and quality assurance, HIV infected participants unaware of their HIV status could have unknowingly infected sexual partners or unborn babies. Although we referred all participants for HIV testing, those in underserved areas had limited or no other options of learning their HIV status. Reducing the time between specimen collection and availability of results in health facilities is an important consideration when planning future surveys.

In Uganda, survey participants cited inconvenience, fear of stigmatization and emotional vulnerability of receiving HIV test results from public facilities as the most common reason for selecting to participate in HBTC [14]. We provided participants the option of collecting results in a health facility in a neighboring cluster and trained counselors to observe strict confidentiality. Similar concerns about confidentiality in KAIS [11] could have negatively impacted collection of results in our survey.

Our analysis has some limitations. In the couple analysis, we only included one primary partner who had taken part in KAIS since we did not collect information on non-KAIS partners. This and the fact that individuals may have accessed HIV testing services in the 6-week waiting period may explain the lower results collection rates in the survey. Despite these limitations, our analysis demonstrates the importance of availing results to survey participants and identifies key gaps in using a facility-based approach that 
will inform planning of future surveys. Our findings also demonstrate the need for countries that conduct household HIV sero-surveys to systematically analyze uptake of test results.

The ultimate goal of returning HIV test results in surveys is to facilitate access to HIV prevention, care and treatment services. Though appropriate referrals were made, the KAIS did not track uptake of referral. In Uganda, lack of HIV care and treatment services for HIV-infected participants was cited as a major drawback of providing HIV test results to survey participants [10]. As HIV surveillance and HIV service delivery become intertwined, surveys that return HIV test results should systematically monitor and evaluate current referral systems. With recent findings supporting earlier treatment [15], HIV-infected persons could seek services earlier. Systematic evaluation of referrals in surveys could help monitor this shift, in contexts where this policy is adopted. Despite the challenges in monitoring survey referrals, data from multiple countries has demonstrated that knowledge of HIV status alone reduces HIV transmission risk by up to $60 \%$; thus HIV testing in surveys provides important HIV prevention benefits [16-18]. The low uptake of HIV test results among young people in urban areas in our analysis coupled with the high prevalence of HIV and risky sexual behaviors among individuals living in urban areas ${ }^{10}$ emphasizes the need to intensify HIV testing and prevention efforts targeting youth in urban areas.

As more countries plan population-based surveys with HIV testing, developing efficient and effective approaches to increase opportunities for participants to learn their HIV status and by extension access available HIV services is critical. With the existence of behavioral and bio-medical interventions proven to prevent HIV infection and lifesaving HIV care and treatment, the benefits of returning HIV test results in surveys are considerable. Other countries may draw important lessons from KAIS 2007 which demonstrated some benefits and limitations of using a facility-based approach to returning HIV test results but also suggested that higher results uptake might occur with a home-based approach.

\section{CONFLICT OF INTEREST}

The authors confirm that this article content has no conflict of interest.

\section{ACKNOWLEDGEMENTS}

We acknowledge the contribution of the 2007 KAIS technical working group, steering committee, and staff from the Kenya Ministry of Health, Kenya National AIDS Control Council, Kenya Medical Research Institute, National Coordinating Agency for Population and Development, U.S. Presidential Emergency Plan for AIDS Relief through the U.S. Centers for Disease Control and Prevention and the United States International Development Agency, the World Health Organization/UNAIDS and the University of California at San Francisco for supporting the planning and implementation of the 2007 Kenya AIDS Indicator Survey. Support for this analysis was provided by the U.S. Presidential Emergency Plan for AIDS Relief (PEPFAR) through the U.S. Centers for Disease Control and Prevention (CDC) in Kenya. Lawrence Marum provided guidance in conceptualization of the manuscript. Vishnu Sneller, Anthony Gichangi and Samuel Mwalili reviewed and gave input on the manuscript. This work is dedicated to the Late Christopher Omollo of the Kenya National Bureau of Statistics for the lead role he played in the planning and implementation of the 2007 KAIS.

We acknowledge the significant contributions of the 2007 KAIS Study Group: Michael Arnold, Godfrey Baltazar, Isaac Baya, John Bore, Rebecca Bunnell, Sufia Dadabhai, Helen Dale, Jared Ichwara, Allen Hightower, Tura Galgalo, Catherine Gichimu, Anthony Gichangi, Reinhard Kaiser, Timothy Kellogg, George Kichamu, Andrea Kim, Evelyn Kim, Samuel Kipruto, George K'opiyo, Ernest Makokha, Barbara Marston, Lawrence Marum, Margaret Mburu, Jonathan Mermin, Joy Mirjahangir, Rex Mpazanje, Ibrahim Mohamed, Patrick Muriithi, James Muttunga, Mary Mwangi, Carol Ngare, Raymond Nyoka, Linus Odawo, Samuel Ogola, Christopher Omolo, Tom Oluoch, Ray Shiraishi, John Wanyungu and Anthony Waruru.

\section{Financial Support}

The 2007 Kenya AIDS Indicator Survey was funded by the U.S. President's Emergency Plan for AIDS Relief through the U.S. Centers for Disease Control and Prevention (CDC), Department of Health and Human Services and the U.S. Agency for International Development (USAID).

\section{DISCLAIMER}

The findings and conclusions in this paper are those of the authors and do not necessarily represent the official views of the U.S. Centers for Disease Control and Prevention or the Kenya Ministries of Health.

\section{REFERENCES}

[1] Diaz T, Carcia-Calleja JM, Ghys PD, Sabin K. Advances and future directions in HIV surveillance in low-and middle-income countries. Curr Opin HIVAIDS 2009; 4: 253-9.

[2] Kishor S. Ethical issues related to population-based surveys with HIV testing. The Demographic and Health Surveys (DHS) Experience. Paper presented at the $2^{\text {nd }}$ Global HIV/AIDS Surveillance meeting, Bangkok, Thailand, March 2-5, 2009.

[3] World Health Organization. Guiding principles on ethical issues in HIV surveillance. UNAIDS/WHO Working Group on Global HIV/AIDS and STI Surveillance. World Health Organization, 2013. Available at: http://apps.who.int/iris/bitstream/10665/90448/ 1/9789241505598_eng.pdf

[4] Pappas G, Hyder AA. Exploring ethical considerations for the use of biological and physiological markers in population-based surveys in less developed countries. Global Health 2005, 1: 16.

[5] Schulte PA, Sweeney MH. Ethical considerations, confidentiality issues, rights of human subjects, and uses of monitoring data in research and regulation. Environ Health Perspect 1995; 103 (Suppl 3): 69-74.

[6] UNAIDS/WHO. Ethics in HIV surveillance Consultation Meeting I: World Health Organization Headquarters, Geneva, Switzerland 2009.

[7] Diaz T. Ethical issues surrounding unlinked anonymous HIV testing surveillance and survey activities in developing countries. Reference available from: URL 'med.brown.edu/.../Background\% 20Ethics $\% 20$ Consult $\% 20$ Concept $\% 20 \mathrm{P}$ '.

[8] Mishra V, Vaessen M, Boerma JT, et al. HIV testing in national population-based surveys: experience from demographic and health surveys. Bull World Health Org 2006; 84 (7): 537-45.

[9] Central Bureau of Statistics (CBS) [Kenya], Ministry of Health $(\mathrm{MOH})$ [Kenya], and ORC Macro. Kenya Demographic and Health Survey 2003, 2004. 
[10] Yoder P, Katahoire AR, Kyaddondo D, Akol Z, Bunnell R, Kaharuza F. Home based HIV testing and counseling in a survey context in Uganda. Calverton, Maryland, USA: ORC Macro 2006.

[11] National AIDS/STI Control Programme (NASCOP), Kenya. 2007 Kenya AIDS Indicator Survey: Final Report 2007. Nairobi, NASCOP. September 2009. Available from: www.nascop.or.ke

[12] Wolff B, Nyanzi B, Katongole G, Ssesanga D, Ruberantwari A, Whitworth J. Evaluation of a home-based voluntary counseling and testing intervention in rural Uganda. Health Policy Plan 2005; 20(2): 109-16.

[13] Dunkle KL, Stephenson R, Karita E, et al. New heterosexually transmitted HIV infections in married or cohabiting couple in urban Zambia and Rwanda: an analysis of survey and clinical data. Lancet 2008; 371: 2183-91.

[14] Kenya National AIDS Control Council. Kenya Analysis of HIV prevention response and modes of transmission study. National AIDS Control Council: Nairobi, Kenya 2009.
[15] Cohen MS, Chen YQ, McCauley M, et al. Prevention of HIV-1 infection with early antiretroviral therapy. N Engl J Med 2011; 11: 365(6): 493-505.

[16] Marks G; Crepaz N, Senterfitt JW, Janssen RS. Meta-Analysis of High-Risk Sexual Behavior in Persons Aware and Unaware They are Infected with HIV in the United States: Implications for HIV Prevention Programs. J Acquir Immune Defic Syndr 2005; 39(4): 446-53.

[17] Bunnell R, Opio A, Musinguzi J, et al. HIV transmission risk behavior among HIV-infected adults in Uganda: results of a nationally representative survey. AIDS 2008; 22(5): 617-24.

[18] Mwangi M, Bunnell R, Nyoka R, et al. Unsafe sex among HIVinfected adults in Kenya: results of a nationally representative survey. J Acquir Immune Defic Syndr 2011; 58(1): 80-8.

(C) Mwangi et al.; Licensee Bentham Open.

This is an open access article licensed under the terms of the Creative Commons Attribution Non-Commercial License (http://creativecommons.org/licenses/by-nc/ 3.0/) which permits unrestricted, non-commercial use, distribution and reproduction in any medium, provided the work is properly cited. 